\title{
Associação entre Depressão, Ansiedade e Qualidade de Vida após Infarto do Miocárdio
}

\author{
Conceição Lemos ${ }^{1}$ \\ Carlos A.M. Gottschall \\ Lucia C. Pellanda \\ Marisa Müller \\ Instituto de Cardiologia do Rio Grande do Sul \\ Fundação Universitária de Cardiologia
}

\begin{abstract}
RESUMO - Este trabalho investigou a freqüência da depressão em indivíduos cardiopatas, com e sem diagnóstico de infarto agudo do miocárdio (IAM), e indivíduos sem diagnóstico de cardiopatia. Trata-se de estudo prospectivo com 168 pacientes divididos em três grupos: 60 internados (diagnóstico de IAM), 49 ambulatoriais (diagnóstico de doença cardiovascular sem infarto) e 59 da população em geral (sem diagnóstico de doença cardiovascular), de ambos os sexos, entre 35 a 65 anos, investigados por meio do Inventário de Depressão de Beck (BDI), do Inventário de Ansiedade de Beck (BAI) e do Teste de Qualidade de Vida (WHOQOL). A prevalência de depressão no grupo IAM foi 48,3\%. As variáveis significativas associadas à depressão foram: história familiar $(\mathrm{RC}=2,82-\mathrm{IC} 95 \%=1,12 ; 7,08-p=0,028)$, domínio psicológico do WHOQOL $(\mathrm{RC}=0,93-\mathrm{IC} 95 \%=0,89 ; 0,98-p=0,006)$ e escore de ansiedade $(\mathrm{RC}=1,08-\mathrm{IC} 95 \%=1,02 ; 1,14-p=0,012)$. Os resultados sugerem que os transtornos de depressão não são desencadeados pelo IAM, mas que estão presentes antes da admissão hospitalar, destacando a importância do rastreamento dos pacientes portadores de doença coronariana crônica.
\end{abstract}

Palavras-chave: infarto agudo do miocárdio; depressão; qualidade de vida; fatores de risco.

\section{Association among Depression, Anxiety, and Quality of Life in Post Acute Myocardial Infarction Patients}

\begin{abstract}
This study investigated the frequency of depression in individuals with cardiovascular disease, with or without an acute myocardial infarction (AMI) diagnosis, and individuals with no cardiovascular disease. It is a prospective study with 168 patients of both genders, from 35 to 65 years old, divided into three groups: 60 inpatients (diagnosed with AMI), 49 outpatients (diagnosed with cardiovascular disease without infarction), and 59 of the general population (without diagnosis of cardiovascular disease). The groups were investigated by applying questionnaires, which included the Beck Depression Inventory (BDI), the Beck Anxiety Inventory (BAI) and the Quality of Life Test (WHOQOL). The prevalence of depression in the AMI group was $48.3 \%$. The variables that remained significantly associated to depression were: family history $(\mathrm{OR}=2.82-95 \% \mathrm{CI}=1.12 ; 7.08-p=0.028)$, WHOQOL psychological domain $(\mathrm{OR}=0.93-95 \% \mathrm{CI}=0.89 ; 0.98-p=0.006)$, and anxiety score $(\mathrm{OR}=1.08-95 \% \mathrm{CI}=1.02 ; 1.14-$ $p=0.012$ ). These results suggest that depression disorders are not triggered post-AMI but can be present before admission to the hospital, which shows the need to investigate people with chronic coronary disease.
\end{abstract}

Keywords: acute myocardial infarction; depression; quality of life; risk factors.

A Organização Mundial da Saúde (OMS) cita a depressão e a doença cardiovascular como duas das condições mais debilitantes e dispendiosas no contexto da saúde, sendo essas doenças crônicas as enfermidades de maior impacto sobre a qualidade de vida do indivíduo (Oliveira Jr, 2005). A depressão é indicada pela OMS como a quinta mais freqüente doença na saúde pública (Tamai, 2003). Ela é nociva à saúde dos pacientes cardiopatas, mostrandose como um agravante fator de risco para o infarto do miocárdio (The American Psychosomatic Society, 2004). Estima-se que a doença arterial coronariana (DAC) e a depressão maior serão em 2020 as duas principais causas de morte (World HeaIth Organization, 2001), devendo

1 Endereço: Unidade de Pesquisa do IC/FUC, Psicologia. Av. Princesa Isabel, 370, Santana. Porto Alegre, RS. CEP 90620-001. E-mail: editoracao-pc@cardiologia.org.br. a possibilidade de comorbidade entre as duas doenças tornar-se preocupante.

Pesquisas recentes indicam que a depressão não tratada adequadamente é considerada tanto um fator de risco para o surgimento do infarto agudo do miocárdio (IAM), como fator de pior prognóstico, aumentando sensivelmente a morbidade e a mortalidade (Tamai, 2003), sendo que de um a três pacientes admitidos em hospitais por doença cardiovascular são acometidos por depressão após o infarto do miocárdio e angina instável (Viola, Kasl, Abramson \& Krumholz, 2001).

Estudos assinalam que 20\% dos pacientes submetidos à coronariografia sofrem de depressão maior. Pacientes acometidos de IAM ou angina instável (AI) também apresentam depressão nos mesmos percentuais (Oliveira Jr., 2005). Investigações multicêntricas chamadas de ENRICHD (Enhancing Recovery in Coronary Heart Disease) referem 


\section{Lemos e cols.}

os transtornos depressivos como presentes em 30 a 50\% dos pacientes que apresentam síndrome coronariana aguda (SCA) (Carney \& cols., 2004).

Mattos, Lougon, Tura e Pereira (2005) investigaram a prevalência de depressão psíquica em pacientes com SCA e a interdependência dos fatores de risco (FR) para doença arterial coronariana (DAC). Dos 135 pacientes internados com SCA (40\% do sexo feminino), com idade média de 61,8 anos, 98 apresentavam diagnóstico de infarto agudo do miocárdio e 37, de angina instável. Depressão foi encontrada em $53,3 \%$ desses pacientes - 28,1\% com depressão leve, $14,1 \%$ com depressão moderada e 11,1\% com depressão grave -, tendo sido mais freqüente em pacientes do sexo feminino. A depressão também esteve presente nas duas associações de maior número de FR: dislipidemia, sedentarismo e sexo ( $p=0,0259)$, e dislipidemia, história familiar e hipertensão arterial sistêmica (HAS) $(p=0,0098)$. Assim, esses autores demonstraram uma alta prevalência de depressão psíquica entre pacientes com SCA com elevado risco relativo de mortalidade e interdependência com outros fatores de risco.

Ainda fazendo referência aos fatores de risco, Peres, Nicolau, Romano e Laranjeira (2005) investigaram em 345 portadores de Síndromes Isquêmicas Miocárdicas Instáveis (SIMI), estratificados por sexo, se características sociodemográficas, diagnóstico clínico, tabagismo, consumo de álcool e ansiedade estariam associados com depressão. Os resultados mostraram que, dentre os pacientes hospitalizados com SIMI, as mulheres, os homens com menos de 50 anos e os ansiosos têm mais chance de apresentarem depressão. Galan (2002) corrobora esses dados mostrando que existem vários fatores de risco na doença cardíaca e que entre eles se destacam os fatores emocionais e as características de personalidade, os quais desempenham um papel de destaque tanto na gênese quanto na recuperação da depressão e do IAM. Galan observou aspectos psicológicos de mulheres infartadas, destacando essa patologia como uma doença grave que interfere na pessoa como um todo, desencadeando reações orgânicas que ameaçam a integridade do ego. Foi observado ainda que o infarto do miocárdio interfere no equilíbrio do indivíduo provocando reações fisiológicas intensas que fogem do seu controle consciente. $\mathrm{O}$ medo da perda do controle provoca reações emocionais nessas pessoas devido à mobilização desencadeada pelo medo da morte, representando uma ameaça. O medo da morte faz a pessoa reavaliar aspectos de sua vida e isso leva com freqüência a processo depressivo.

$\mathrm{O}$ estudo ENRICHD indica que apenas $10 \%$ das pessoas com depressão pós IAM são diagnosticadas como tal (Carney \& cols., 2004). Portanto, o estudo da prevalência deste agravo em pacientes portadores de IAM em nosso meio pode fornecer elementos para um melhor planejamento do atendimento desses, enfatizando a importância do diagnóstico adequado. Carney (2004) e Fleck (2000) fazem referência ao impacto emocional provocado pela depressão pós-infarto e pelos inúmeros danos à qualidade de vida, sendo a qualidade de vida considerada em um sentido mais amplo do que de controle de sintomas, diminuição da mortalidade ou aumento da expectativa de vida.

Face ao exposto, este trabalho tem como objetivo investigar a freqüência da depressão pós-infarto agudo do miocárdio durante o período de hospitalização em uma instituição de referência em Cardiologia, comparando esta freqüência com a de pacientes cardiopatas ambulatoriais e com uma população sem diagnóstico de cardiopatia.

\section{Participantes}

\section{Método}

Este estudo envolveu um grupo de expostos e dois grupos controle, tendo sido realizado no Instituto de Cardiologia do Rio Grande do Sul (IC/FUC), um centro de referência para cardiologia. Neste estudo, foram avaliados 224 indivíduos pareados por idade e sexo. O estudo foi aprovado pelo Comitê de Ética do hospital e realizado no período de janeiro de 2004 a fevereiro de 2005 . Todos os participantes assinaram o Termo de Consentimento Livre e Esclarecido.

Foram incluídos no estudo 168 indivíduos, entre 35 e 65 anos, de ambos os gêneros, dos quais 60 pertenciam ao grupo 'internação' (pacientes internados no IC/FUC com diagnóstico de IAM durante o período do estudo), 49 ao grupo 'controle ambulatorial' (consulentes do ambulatório com diagnóstico de doenças cardiovasculares, mas sem diagnóstico de IAM), e 59 ao grupo ‘controle externo' (indivíduos de uma população não selecionada, sem diagnóstico de doença cardiovascular (DCV).

Foram excluídos 56 indivíduos com diagnóstico de depressão por estarem em uso de antidepressivos, em tratamento psicoterápico ou por apresentarem outros transtornos psiquiátricos que impediam a compreensão e a comunicação, sendo $15(20 \%)$ do grupo internação, 25 (34\%) do grupo controle ambulatorial e 16 (21,3\%) do grupo controle externo.

\section{Procedimento}

Os indivíduos incluídos no estudo foram divididos em três grupos:

- Grupo Internação: indivíduos internados com diagnóstico de IAM registrado nos prontuários, estabelecido pelo cardiologista responsável e validado pelos autores quando dois dos seguintes critérios estivessem presentes: (a) história de desconforto torácico prolongado (>20 min), não aliviado por nitrato sublingual; (b) alteração na evolução do eletrocardiograma consistente com necrose (desenvolvimento de ondas Q) e/ou elevação do segmento ST > 1 mm; (c) mensurações seriadas tardias dos marcadores creatinoquinase total (CK) e sua fração MB (CK-MB);

- Grupo Controle Ambulatorial: indivíduos sem IAM, consulentes do ambulatório de cardiopatia isquêmica, com diagnóstico obtido por meio de teste de esforço realizado em esteira ergométrica, com cinecoronariografia e ventriculografia registrados nos prontuários dos mesmos;

- Grupo Controle Externo: indivíduos oriundos da mesma comunidade que os dois grupos anteriores e sem diagnóstico ou antecedente de qualquer doença cardiovascular.

\section{Instrumentos de avaliação}

Os 168 indivíduos selecionados foram entrevistados e avaliados pelo Inventário de Ansiedade de Beck (BAI), Inventário de Depressão de Beck (BDI), WHOQOL (ins- 
trumento que analisa a qualidade de vida) e Ficha SócioDemográfica (Fleck \& cols., 2000; Gorenstein, Andrade \& Antonio, 1999), por um investigador cego quanto ao diagnóstico cardiovascular. Os pacientes de cada grupo (internação, controle ambulatorial e controle externo) foram divididos em dois subgrupos: deprimidos (escore de Beck >9) e os não deprimidos (escore de Beck<10).

\section{Análise estatística}

A análise foi realizada por meio de estatística descritiva (média, mediana, desvio padrão, mínimo, máximo, freqüências e percentuais), do teste $t$ de Student, da análise de variância (ANOVA) e teste Qui-Quadrado, com nível de significância de 5\%. Adicionalmente, foi utilizado um modelo de regressão logística que incluiu as variáveis $\operatorname{com} p<0,20$ ou consideradas clinicamente significativas de acordo com o modelo teórico.

\section{Resultados}

As características demográficas e clínicas dos 168 participantes, bem como os resultados dos testes BAI, BDI e WHOQOL (Fleck \& cols., 2000; Gorenstein \& cols., 1999) com seus domínios, estão descritos na Tabela 1.

Com relação às características clínicas que apresentaram resultados estatisticamente significativos entre os grupos foram observados os seguintes resultados. O tabagismo apresentou uma incidência significativa de $75 \%$ no grupo internação e $42,9 \%$ e $23,7 \%$ nos grupos controle ambulatorial e controle externo, respectivamente $(p<0,001)$. O BAI foi maior para os participantes do grupo internação $(13,0 \pm 10,6)$ e menor para aqueles do grupo controle externo $(7,2 \pm 7,8)$. Em relação ao WHOQOL, o domínio físico apresentou valores mais baixos no grupo controle ambulatorial. A prevalência de depressão

Tabela 1. Características clinico-demográficas da amostra.

\begin{tabular}{|c|c|c|c|c|}
\hline \multirow{2}{*}{ Variáveis } & \multicolumn{3}{|c|}{ Grupos } & \multirow[b]{2}{*}{$p$} \\
\hline & $\begin{array}{c}\text { Internação } \\
(n=60)\end{array}$ & $\begin{array}{c}\text { Controle ambulatorial } \\
(n=49)\end{array}$ & $\begin{array}{c}\text { Controle externo } \\
(\mathrm{n}=59)\end{array}$ & \\
\hline Sexo masculino & $48(80,0)$ & $34(69,4)$ & $40(67,8)$ & $0,274 *$ \\
\hline Idade & $50,7 \pm 7,4$ & $51,1 \pm 8,5$ & $50,0 \pm 7,5$ & $0,754 * *$ \\
\hline Estado civil & & & & $0,068^{*}$ \\
\hline Casado & $43(71,7)$ & $36(73,5)$ & $37(62,7)$ & \\
\hline Solteiro & $3(5,0)$ & $6(12,2)$ & $13(22,0)$ & \\
\hline Divorciado / viúvo & $14(23,3)$ & $7(14,3)$ & $9(15,3)$ & \\
\hline Tabagismo & $45(75,0)$ & $21(42,9)$ & $14(23,7)$ & $<0,001 *$ \\
\hline Diabete & $9(15,0)$ & $3(6,1)$ & $3(5,1)$ & $0,118^{*}$ \\
\hline Obesidade & $15(25,0)$ & $12(24,5)$ & $11(18,6)$ & $0,662 *$ \\
\hline Hipertensão & $27(45,0)$ & $27(55,1)$ & $13(22,0)$ & $0,001 *$ \\
\hline Hipercolesterolemia & $17(28,3)$ & $17(34,7)$ & $14(23,7)$ & $0,454^{*}$ \\
\hline História familiar IAM & $43(71,7)$ & $32(65,3)$ & $31(52,5)$ & $0,090^{*}$ \\
\hline Alcoolismo & $11(18,3)$ & $5(10,2)$ & $6(10,2)$ & $0,325^{*}$ \\
\hline BAI & $13,0 \pm 10,6$ & $11,3 \pm 9,3$ & $7,2 \pm 7,8$ & $0,003 * *$ \\
\hline $\mathrm{BDI} \geq 10$ & $29(48,3)$ & $26(53,1)$ & $12(20,3)$ & $0,001 *$ \\
\hline WHOQOL - Domínio Físico & $65,6 \pm 18,0$ & $63,0 \pm 14,1$ & $73,6 \pm 15,3$ & $0,002 * *$ \\
\hline WHOQOL - Domínio Psicológico & $70,8 \pm 14,6$ & $70,5 \pm 12,9$ & $72,0 \pm 11,6$ & $0,809 * *$ \\
\hline WHOQOL - Domínio Relações Sociais & $76,3 \pm 15,4$ & $75,3 \pm 15,2$ & $74,4 \pm 17,4$ & $0,827 * *$ \\
\hline WHOQOL - Domínio Meio Ambiente & $63,2 \pm 15,6$ & $59,9 \pm 11,5$ & $63,5 \pm 14,2$ & $0,351 * *$ \\
\hline WHOQOL - Overall & $69,8 \pm 19,4$ & $66,6 \pm 17,9$ & $72,0 \pm 17,6$ & $0,309 * *$ \\
\hline
\end{tabular}

Notas: Valores apresentados como freqüência absoluta (percentual) ou média \pm desvio-padrão

BAI: Inventário de Ansiedade de Beck; BDI: Inventário de Depressão de Beck

* Teste de $\chi^{2}$ de Pearson. ** Análise de variância (ANOVA), seguido do teste de comparações múltiplas de Tukey 
(BDI $>10)$ foi maior no grupo controle ambulatorial, com $53,1 \%$, e menor no grupo controle externo, com $20,3 \%$.

A comparação das características demográficas e clínicas entre os pacientes com depressão e sem depressão estão apresentadas na Tabela 2. Os pacientes com depressão, quando comparados aos pacientes sem depressão, apresentaram uma maior proporção de tabagismo e história familiar de IAM, maiores escores no BAI e menores escores nos quatro domínios e escore geral do WHOQOL.

Para avaliar a interdependência de cada um dos fatores de risco clássicos para IAM e considerando a depressão como desfecho, utilizou-se a análise de regressão logística múltipla das características clínicas, qualidade de vida, ansiedade e os grupos avaliados, conforme indicado na Tabela 3. As variáveis que permaneceram associadas, com significância estatística, à depressão no modelo multivariado foram: história familiar de IAM - risco clássico - $(\mathrm{RC}=$ $2,82$ - IC95\%=1,12;7,08 $-p=0,028)$, domínio psicológico do WHOQOL $(\mathrm{RC}=0,93$ - IC95\%=0,89;0,98 - $p=0,006)$ e escore de ansiedade $(\mathrm{RC}=1,08$ - $\mathrm{IC} 95 \%=1,02 ; 1,14-p=0,012)$. $\mathrm{O}$ grupo controle ambulatorial apresentou maiores índices de depressão quando comparado com o grupo controle externo $(\mathrm{RC}=3,24-\mathrm{IC} 95 \%=1,08 ; 9,75-p=0,036)$.

\section{Discussão}

No presente estudo, a freqüência de depressão foi maior no grupo controle ambulatorial, cujos pacientes estavam sob acompanhamento médico. Estudos corroboram esse resultado mostrando que a depressão não é uma conseqüência direta do infarto do miocárdio, mas está presente antes da admissão hospitalar (Mattos \& cols., 2005). Lauzon e cols. (2003), por outro lado, em seus estudos com pacientes de IAM, não encontraram escores significativos no BDI na sua amostra. Esses achados demonstram que a depressão é subdiagnosticada e subtratada antes e após o IAM. O fato de subestimar a depressão é explicado pela falta do devido apreço aos sintomas depressivos, interpretando-os como transitórios e "naturais" a um quadro clínico, bem como aos precários conhecimentos dos fatores de risco relacionados ao desenvolvimento da depressão após o infarto do miocárdio.

Tabela 2. Comparação das características demográficas e clínicas entre os pacientes com e sem depressão.

\begin{tabular}{|c|c|c|c|}
\hline \multirow{2}{*}{ Variáveis } & \multicolumn{2}{|c|}{ BDI } & \multirow{2}{*}{$p$} \\
\hline & $\geq 10(n=67)$ & $<10(n=101)$ & \\
\hline Sexo masculino & $45(67,2)$ & $77(76,2)$ & $0,265^{*}$ \\
\hline Idade & $50,5 \pm 8,3$ & $50,6 \pm 7,4$ & $0,912 * *$ \\
\hline Estado civil & & & $0,843 \#$ \\
\hline Casado & $45(67,2)$ & $71(70,3)$ & \\
\hline Solteiro & $10(14,9)$ & $12(11,9)$ & \\
\hline Divorciado / viúvo & $12(17,9)$ & $18(17,8)$ & \\
\hline Tabagismo & $40(59,7)$ & $40(39,6)$ & $0,017 *$ \\
\hline Diabete & $7(10,4)$ & $8(7,9)$ & $0,775^{*}$ \\
\hline Obesidade & $13(19,4)$ & $25(24,8)$ & $0,533^{*}$ \\
\hline Hipertensão & $33(49,3)$ & $34(33,7)$ & $0,063^{*}$ \\
\hline Hipercolesterolemia & $23(34,3)$ & $25(24,8)$ & $0,242 *$ \\
\hline História Familiar IAM & $49(73,1)$ & $57(56,4)$ & $0,042 *$ \\
\hline Alcoolismo & $11(16,4)$ & $11(10,9)$ & 0,420 \\
\hline BAI & $15,7 \pm 11,0$ & $7,0 \pm 6,5$ & $<0,001 * *$ \\
\hline WHOQOL - Domínio Físico & $58,8 \pm 16,4$ & $73,5 \pm 13,9$ & $<0,001 * *$ \\
\hline WHOQOL - Domínio Psicológico & $63,7 \pm 13,6$ & $76,1 \pm 10,0$ & $<0,001 * *$ \\
\hline WHOQOL - Domínio Relações Sociais & $71,1 \pm 18,7$ & $78,1 \pm 13,3$ & $0,009^{* *}$ \\
\hline WHOQOL - Domínio Meio Ambiente & $55,6 \pm 12,9$ & $66,8 \pm 13,0$ & $<0,001 * *$ \\
\hline WHOQOL - Overall & $62,7 \pm 20,4$ & $74,3 \pm 15,4$ & $<0,001 * *$ \\
\hline
\end{tabular}


Tabela 3. Análise de regressão logística simples e múltipla das características clínicas, qualidade de vida, ansiedade e os grupos avaliados em relação à depressão.

\begin{tabular}{|c|c|c|c|c|}
\hline \multirow{2}{*}{ Variáveis } & \multicolumn{2}{|c|}{ Análise bruta } & \multicolumn{2}{|c|}{ Análise ajustada } \\
\hline & $\operatorname{RC}\left(\mathrm{IC}_{95 \%}\right)$ & $p^{*}$ & $\mathrm{RC}\left(\mathrm{IC}_{95 \%}\right)$ & $p^{*}$ \\
\hline Tabagismo & $2,3(1,2 ; 4,2)$ & 0,011 & $1,51(0,58 ; 3,94)$ & 0,403 \\
\hline Hipertensão & $1,9(1,01 ; 3,6)$ & 0,044 & $0,90(0,37 ; 2,20)$ & 0,824 \\
\hline História familiar IAM & $2,1(1,1 ; 4,1)$ & 0,029 & $2,82(1,12 ; 7,08)$ & 0,028 \\
\hline WHOQOL - Domínio Físico & $0,94(0,92 ; 0,96)$ & $<0,001$ & $0,98(0,95 ; 1,01)$ & 0,207 \\
\hline WHOQOL - Domínio Psicológico & $0,91(0,88 ; 0,94)$ & $<0,001$ & $0,93(0,89 ; 0,98)$ & 0,006 \\
\hline WHOQOL - Domínio Relações Sociais & $0,97(0,95 ; 0,99)$ & 0,007 & $1,00(0,97 ; 1,03)$ & 0,912 \\
\hline WHOQOL - Domínio Meio Ambiente & $0,94(0,91 ; 0,96)$ & $<0,001$ & $0,97(0,93 ; 1,01)$ & 0,100 \\
\hline WHOQOL - Overal & $0,96(0,95 ; 0,98)$ & $<0,001$ & $1,02(0,99 ; 1,05)$ & 0,280 \\
\hline BAI & $1,13(1,08 ; 1,18)$ & $<0,001$ & $1,08(1,02 ; 1,14)$ & 0,012 \\
\hline \multicolumn{5}{|l|}{ Grupo } \\
\hline Internação & $3,7(1,6 ; 8,2)$ & 0,001 & $2,21(0,67 ; 7,32)$ & 0,193 \\
\hline Controle ambulatorial & $4,4(1,9 ; 10,3)$ & 0,002 & $3,24(1,08 ; 9,75)$ & 0,036 \\
\hline Controle externo & 1,0 & & 1,0 & \\
\hline
\end{tabular}

$\mathrm{n}=168$; RC: Razão de Chances; BAI: Inventário de Ansiedade de Beck;

* Teste de $\chi^{2}$ de Wald

Outro estudo, no qual pacientes cardiopatas com história de sintomas depressivos foram comparados com pacientes não deprimidos, concluiu que o risco relativo para os depressivos sofrerem um evento coronariano é comparável ao risco da obesidade, sendo um pouco mais baixo que o hábito de fumar (The American Psychosomatic Society, 2004).

De acordo com Gorenstein e cols. (1999), o paciente, diante dos sintomas pós IAM, vivencia uma situação de ansiedade. No presente estudo, a ansiedade foi verificada pelo BAI, tendo sido verificado que está mais presente no grupo internação. Esses dados foram observados no momento da entrevista, quando os pacientes relatavam sua experiência frente ao evento, felizes por terem sobrevivido, e pensando em reformular suas vidas. Os sintomas de ansiedade nesse grupo foram altos em virtude do BAI verificar sintomas físicos, e esses pacientes estarem fragilizados pelo infarto (Gorenstein \& cols., 1999).

Para o paciente, o infarto representa uma ameaça de morte, mostrando-se como um obstáculo na sua caminhada de vida e alterando sua visão de mundo. Nesse momento, o paciente vivencia um alto grau de ansiedade, como também sua auto-estima fica alterada devido ao infarto (Oliveira Jr, 2005). Assim, é possível especular que os pacientes que sofreram um IAM tiveram a via do corpo como expressão da depressão e da ansiedade, sendo a primeira mais evidente antes do evento.

Nosso estudo mostrou, a partir da análise da qualidade de vida efetuada por meio do WHOQOL, que houve significância estatística no item do domínio físico, representando um escore maior no grupo internação, o que torna evidente a fragilidade da qualidade de vida. No mesmo grupo, a ansiedade corrobora resultados pertinentes ao grau de limitação e desconforto à vida do paciente. Nessa linha, Fleck e cols. (2000) fazem referência à crescente preocupação, não só com a freqüência e a severidade das doenças, como também com o impacto dessas, comprometendo as atividades diárias do paciente.

Os resultados de Fleck e cols. (2000) são consistentes com aqueles aqui relatados, no que se refere à ansiedade pós IAM e aos resquícios por ela deixados. Os resultados aqui obtidos também estão de acordo com o trabalho de Pereze cols. (2005), os quais afirmam que pacientes hospitalizados com síndromes isquêmicas miocárdicas instáveis e com maiores níveis de ansiedade têm maior probabilidade de apresentarem depressão.

No presente trabalho também se observou associação significativa entre os escores de depressão e ansiedade. Os pacientes deprimidos apresentaram uma evidência significativa de comprometimento em todos os domínios do WHOQOL, o que demonstra o prejuízo da qualidade de vida. Esse prejuízo pode estar associado à intensa presença de fatores de risco nessa população, já que se verificou entre os pacientes estudados a existência de uma associação significativa entre o diagnóstico de depressão e a presença de fatores de risco.

Em suma, o presente trabalho sugere que os transtornos de depressão não são desencadeados pelo IAM, mas que estavam presentes antes da admissão hospitalar, enfatizando 


\section{Lemos e cols.}

a importância da detecção precoce e do diagnóstico adequado para o planejamento de intervenções efetivas nesta condição extremamente prevalente.

\section{Considerações Finais}

Algumas limitações do estudo devem ser consideradas. Por se tratar de um estudo observacional, é possível que fatores de confusão não avaliados interferiram nos resultados. O delineamento de exposto-controle também não permite o estabelecimento de uma relação temporal clara entre os eventos. Estudos adicionais são necessários para avaliar prospectivamente o papel da depressão como fator de risco ou desencadeante de desfechos clínicos cardiovasculares.

\section{Referências}

Carney, R. M. \& Freedland, K. E. (2005). Depression and coronary heart disease: more pieces of the puzzle. American Journal of Psyquiatry, 164, 1307-1309.

Carney, R. M., Blumenthal, J. A., Fredland, K. E., Youngblood, M., Veith, R. C., Burg, M.M., Cornell, C., Saab, P. G., Kaufmann, P. G., Czajkowski, S. M., Jaffe, A. S. - ENRICHD Investigators (2004). Depression and late mortality after myocardial infarction in the Enhancing Recovery in Coronary Heart Disease (ENRICHD) study. Psychosomatic Medicine, 66, 466-474.

Fleck, M. P. A., Louzada, S., Xavier, M., Chachamovich, E., Vieira, G., Santos, L. \& Pinzon, V. (2000). Aplicação da versão em português do instrumento de avaliação de qualidade de vida da Organização Mundial da Saúde WHOQOL-100/Bref. Revista de Saúde Pública, 2, 178-183.

Frasure-Smith, N. \& Lesperance, F. (2005). Reflections on depression as a cardiac risk factor. Psychosamatic Medicine, 67(Supp/I), 19-25.

Galan H. D S. (2002). Um estudo psicológico sobre o infarto do miocárdio em mulheres artigo. Dissertação de Mestrado, Pontifícia Universidade Católica de São Paulo, São Paulo.

Gorenstein, C., Andrade, H. S. G. \& Antonio, L. Z. (1999). Escalas de avaliação clínica em psiquiatria e psicofarmacologia. Revista de Psiquiatria Clínica, 25, 89-143.
The American Psychosomatic Society (2004). INTERHEART study: Intersection between behavioral and general medicine. Psychosomatic Medicine, 66, 797-798.

Lauzon, C. E., Beck, C. A., Huynh, D. D., Racine, N., Caringnam, S. \& Diodati, J. G. (2003) Depression and prognosis following hospital admission because of acute myocardial infarction. Canadian Medical Association Journal, 168, 547-552.

Mattos, M. A., Lougon, M., Tura, B. R. \& Pereira, B. B. (2005). Depressão e Síndrome Isquêmica Coronariana Aguda. Revista da Sociedade de Cardiologia do Estado de São Paulo, 18, 288-294.

Oliveira Jr, W. (2005). Relação médico-paciente em Cardiologia: um olhar psicossomático. Revista de Medicina Psicossomática Sul Mineira, 4, 41-46.

Peres, G. H., Nicolau, J. C., Romano, B. W. \& Laranjeira, R. (2005). Depressão e síndromes isquêmicas miocárdicas instáveis: diferenças entre homens e mulheres. Arquivos Brasileiros de Cardiologia, 85, 319-322.

Tamai, S. (2003). Depressão e infarto do miocárdio. Tese de Doutorado, Departamento de Psiquiatria e Psicologia Médica da Faculdade de Ciências Médicas da Santa Casa de São Paulo, São Paulo.

Viola, V., Kasl, S. V., Abramson, J. \& Krumholz, H. M. (2001). Depressive symptoms and risk of functional decline and death in patients with heart failure. Journal of Abmerican College of Cardiology, 38, 199-205.

World HeaIth Organization - WHO (2001). World Health Report 2001 - Mental Health: New Understanding, New Hope, 2, 29-30. 